KYUNGPOOK Math. J. 53(2013), 615-624

http://dx.doi.org/10.5666/KMJ.2013.53.4.615

\title{
Majorization Properties for Subclasses of Analytic p-Valent Functions Defined by Convolution
}

Rabha Mohamed El-AshwaH*

Department of Mathematics, Faculty of Science, Damietta University, New Damietta 34517, Egypt

e-mail : r_elashwah@yahoo.com

Mohamed Kamal Aouf

Department of Mathematics, Mansoura University, Mansoura 35516, Egypt

e-mail : mkaouf127@yahoo.com

Abstract. The object of the present paper is to investigate the majorization properties of certain subclasses of analytic p-valent functions defined by convolution.

\section{Introduction}

Let $f$ and $g$ be analytic in the open unit $\operatorname{disc} U=\{z \in \mathbb{C}:|z|<1\}$. We say that $f$ is majorized by $g$ in $U$ (see [7]) and write

$$
f(z)<<g(z) \quad(z \in U)
$$

if there exists a function $\varphi$, analytic in $U$ such that

$$
|\varphi(z)|<1 \quad \text { and } \quad f(z)=\varphi(z) g(z) \quad(z \in U) .
$$

It may be noted that (1.1) is closely related to the concept of quasi-subordination between analytic functions.

If $f$ and $g$ are analytic functions in $U$, we say that $f$ is subordinate to $g$, written symbolically as $f(z) \prec g(z)$ if there exists a Schwarz function $w$, which (by definition) is analytic in $U$ with $w(0)=0$ and $|w(z)|<1$ for all $z \in U$, such that $f(z)=g(w(z)), z \in U$. Furthermore, if the function $g$ is univalent in $U$, then we have the following equivalence, (see $[8$, p.4]):

$$
f(z) \prec g(z) \Leftrightarrow f(0)=g(0) \text { and } f(U) \subset g(U) .
$$

* Corresponding Author.

Received January 15, 2011; accepted December 22, 2012.

2010 Mathematics Subject Classification: 30C45.

Key words and phrases: Analytic functions, convolution, majorization. 
Let $A(p)$ denote the class of functions of the form:

$$
f(z)=z^{p}+\sum_{k=p+1}^{\infty} a_{k} z^{k} \quad(p \in \mathbb{N}=\{1,2, \ldots\}),
$$

which are analytic and p-valent in the open unit disc $U$. Also let $A=A(1)$.

For functions $f_{i} \in A(p)(i=1,2)$ given by

$$
f_{i}(z)=z^{p}+\sum_{k=p+1}^{\infty} a_{k, i} z^{k} \quad(i=1,2 ; p \in \mathbb{N}),
$$

we define the Hadamard product (or convolution) of $f_{1}$ and $f_{2}$ by

$$
\left(f_{1} * f_{2}\right)(z)=z^{p}+\sum_{k=p+1}^{\infty} a_{k, 1} a_{k, 2} z^{k}=\left(f_{2} * f_{1}\right)(z) .
$$

For $h(z)$ given by

$$
h(z)=z^{p}+\sum_{k=p+1}^{\infty} \mu_{k} z^{k} \quad\left(\mu_{k} \geq 0\right),
$$

then a function $f \in A(p)$ is said to be in the class $S_{p}^{j}(h ; \gamma)$ of p-valent starlike functions of complex order $\gamma \in \mathbb{C}^{*}=\mathbb{C} \backslash\{0\}$ in $U$, if and only if

$$
\operatorname{Re}\left\{1+\frac{1}{\gamma}\left(\frac{z(f * h)^{(j+1)}(z)}{(f * h)^{(j)}(z)}-p+j\right)\right\}>0
$$

$$
\left(z \in U ; p \in \mathbb{N} ; j \in \mathbb{N}_{0}=\mathbb{N} \cup\{0\} ; \gamma \in \mathbb{C}^{*} ;|2 \gamma-p+j|<(p-j)\right) .
$$

Furthermore, a function $f \in A(p)$ is said to be in the class $K_{p}^{j}(h, \gamma)$ of p-valent convex functions of complex order $\gamma \in \mathbb{C}^{*}$ in $U$, if and only if

$$
\begin{gathered}
\operatorname{Re}\left\{1+\frac{1}{\gamma}\left(\frac{z(f * h)^{(j+2)}(z)}{(f * h)^{(j+1)}(z)}-p+j+1\right)\right\}>0 \\
\left(z \in U ; p \in \mathbb{N} ; j \in \mathbb{N}_{0} ; \gamma \in \mathbb{C}^{*} ;|\gamma-p+j|<(p-j)\right) .
\end{gathered}
$$

From (1.6) and (1.7) it follows that:

$$
\text { (i) } S_{1}^{0}\left(\frac{z}{1-z} ; \gamma\right)=S(\gamma) \text { and } K_{1}^{0}\left(\frac{z}{1-z} ; \gamma\right)=K(\gamma)\left(\gamma \in \mathbb{C}^{*}\right)
$$

The class $S(\gamma)$ was introduced by Nasr and Aouf [9], and the class $K(\gamma)$ was introduced and studied by Nasr and Aouf [9] and Wiatrowski [13].

(ii) $S_{p}^{0}\left(\frac{z^{p}}{1-z} ; p-\alpha\right)=S_{p}^{*}(\alpha)$ and $K_{p}^{0}\left(\frac{z^{p}}{1-z} ; p-\alpha\right)=K_{p}(\alpha) \quad(0 \leq \alpha<p)$. 
The class $S_{p}^{*}(\alpha)$ was introduced by Patil and Thakare [12], and the class $K_{p}(\alpha)$ was introduced by Owa [11]. We note that

$$
S_{p}^{*}(\alpha) \subseteq S_{p}^{*}(0)=S_{p}^{*} \text { and } K_{p}(\alpha) \subseteq K_{p}(0)=K_{p} \quad(0 \leq \alpha<p),
$$

where $S_{p}^{*}$ and $K_{p}$ denote the subclasses of $A(p)$ consisting of functions which are $p$-valent starlike in $U$ and $p$-valent convex in $U$, respectively (see Goodman [5]).

Definition 1.1. Let $-1 \leq B<A \leq 1, p \in \mathbb{N}, j \in \mathbb{N}_{0}, \gamma \in \mathbb{C}^{*},|\gamma(A-B)+(p-j) B|<$ $(p-j), f \in A(p)$ and $h(z)$ is given by (1.5). Then $f \in S_{p}^{j}(h ; \gamma ; A, B)$, the class of p-valent functions of complex order $\gamma$ in $U$ if and only if

$$
\left\{1+\frac{1}{\gamma}\left(\frac{z(f * h)^{(j+1)}(z)}{(f * h)^{(j)}(z)}-p+j\right)\right\} \prec \frac{1+A z}{1+B z} .
$$

Clearly, we have the following relationships:

(i) $S_{p}^{j}(h ; \gamma ; 1,-1)=S_{p}^{j}(h ; \gamma)$;

(ii) $S_{p}^{j}\left(\frac{z^{p}}{1-z} ; \gamma\right)=S_{p}^{j}(\gamma)$.

We shall need the following lemma.

Lemma 1.2([1]). Let $\gamma \in \mathbb{C}^{*}$ and $f \in K_{p}^{j}(\gamma)$. Then $f \in S_{p}^{j}\left(\frac{1}{2} \gamma\right)$, that is,

$$
K_{p}^{j}(\gamma) \subset S_{p}^{j}\left(\frac{1}{2} \gamma\right)\left(\gamma \in \mathbb{C}^{*}\right)
$$

A majorization problem for the classes $S(\gamma)$ and $K(\gamma)$ has recently been investigated by Altintas et al. [2]. Also, majorization problem for the classes $S^{*}=S^{*}(0)$ and $K=K(0)$ has been investigated by MacGregor [7]. In this paper we investigate majorization problem for the class $S_{p}^{j}(h, \gamma ; A, B)$ and some related subclasses.

\section{Main Results}

Unless otherwise mentioned we shall assume throughout the paper that, $-1 \leq$ $B<A \leq 1, \gamma \in \mathbb{C}^{*}, p \in \mathbb{N}, j \in \mathbb{N}_{0}$ and $h(z)$ is given by (1.5).

Theorem 2.1. Let the function $f \in A(p)$ and suppose that $(g * h) \in S_{p}^{j}(h, \gamma ; A, B)$. If $(f * h)^{(j)}(z)$ is majorized by $(g * h)^{(j)}(z)$ in $U$, then

$$
\left|(f * h)^{(j+1)}(z)\right| \leq\left|(g * h)^{(j+1)}(z)\right| \quad\left(|z|<r_{0}\right),
$$

where $r_{0}=r_{0}(p, \gamma, j, A, B)$ is the smallest positive root of the equation

$$
|\gamma(A-B)+(p-j) B| r^{3}-[2|B|+(p-j)] r^{2}-
$$

$$
[2+|\gamma(A-B)+(p-j) B|] r+(p-j)=0 .
$$


Proof. Since $(g * h) \in S_{p}^{j}(h, \gamma ; A, B)$, we find from (1.8) that

$$
1+\frac{1}{\gamma}\left(\frac{z(g * h)^{(j+1)}(z)}{(g * h)^{(j)}(z)}-p+j\right)=\frac{1+A w(z)}{1+B w(z)},
$$

where $w$ is analytic in $U$ with $w(0)=0$ and $|w(z)|<1(z \in U)$. From (2.3), we have

$$
\frac{z(g * h)^{(j+1)}(z)}{(g * h)^{(j)}(z)}=\frac{(p-j)+(\gamma(A-B)+(p-j) B) w(z)}{1+B w(z)} .
$$

From (2.4), we have

$$
\left|(g * h)^{(j)}(z)\right| \leq \frac{(1+|B||z|)|z|}{(p-j)-|\gamma(A-B)+(p-j) B||z|}\left|(g * h)^{(j+1)}(z)\right| .
$$

Next, since $(f * h)^{(j)}(z)$ is majorized by $(g * h)^{(j)}(z)$ in $U$, from (1.2), we have

$$
(f * h)^{(j)}(z)=\varphi(z)(g * h)^{(j)}(z) .
$$

Differentiating (2.6) with respect to $z$, we have

$$
(f * h)^{(j+1)}(z)=\varphi^{\prime}(z)(g * h)^{(j)}(z)+\varphi(z)(g * h)^{(j+1)}(z) .
$$

Thus, by noting that $\varphi(z)$ satisfies the inequality (see [10]),

$$
\left|\varphi^{\prime}(z)\right| \leq \frac{1-|\varphi(z)|^{2}}{1-|z|^{2}}(z \in U),
$$

using (2.5) and (2.8), in (2.7), we have

$$
\begin{aligned}
& \left|(f * h)^{(j+1)}(z)\right| \leq \\
& \left(|\varphi(z)|+\frac{1-|\varphi(z)|^{2}}{1-|z|^{2}} \cdot \frac{(1+|B||z|)|z|}{(p-j)-|\gamma(A-B)+(p-j) B||z|}\right)\left|(g * h)^{(j+1)}(z)\right|,
\end{aligned}
$$

which upon setting

$$
|z|=r \text { and }|\varphi(z)|=\rho(0 \leq \rho \leq 1),
$$

leads us to the inequality

$$
\begin{aligned}
& \left|(f * h)^{(j+1)}(z)\right| \leq \\
& \frac{\Theta(\rho)}{\left(1-r^{2}\right)((p-j)-|\gamma(A-B)+(p-j) B| r)}\left|(g * h)^{(j+1)}(z)\right|,
\end{aligned}
$$


where

$$
\begin{aligned}
\Theta(\rho)= & -r(1+|B| r) \rho^{2}+\left(1-r^{2}\right)[(p-j)-|\gamma(A-B)+(p-j) B| r] \rho \\
& +r(1+|B| r)
\end{aligned}
$$

takes its maximum value at $\rho=1$, with $r_{0}=r_{0}(p, j, \gamma, A, B)$, where $r_{0}(p, j, \gamma, A, B)$ is the smallest positive root of $(2.2)$. Therefore the function $\Phi(\rho)$ defined by

$$
\begin{aligned}
\Phi(\rho)= & -\sigma(1+|B| \sigma) \rho^{2}+\left(1-\sigma^{2}\right)[(p-j)-|\gamma(A-B)+(p-j) B| \sigma] \rho \\
& +\sigma(1+|B| \sigma)
\end{aligned}
$$

is an increasing function on the interval $0 \leq \rho \leq 1$, so that

$$
\begin{gathered}
\Phi(\rho) \leq \Phi(1)=\left(1-\sigma^{2}\right)[(p-j)-|\gamma(A-B)+(p-j) B| \sigma] \\
\left(0 \leq \rho \leq 1 ; 0 \leq \sigma \leq r_{0}(p, j, \gamma, A, B)\right) .
\end{gathered}
$$

Hence upon setting $\rho=1$ in (2.11), we conclude that (2.1) holds true for $|z| \leq r_{0}=$ $r_{0}(p, \gamma, j, A, B)$, where $r_{0}(p, \gamma, j, A, B)$, is the smallest positive root of $(2.2)$. This completes the proof of Theorem 2.1 .

Putting $A=1$ and $B=-1$ in Theorem 2.1, we obtain the following result.

Corollary 2.2. Let the function $f \in A(p)$ and suppose that $g \in S_{p}^{j}(h ; \gamma)$. If $(f *$ $h)^{(j)}(z)$ is majorized by $(g * h)^{(j)}(z)$ in $U$, then

$$
\left|(f * h)^{(j+1)}(z)\right| \leq\left|(g * h)^{(j+1)}(z)\right|\left(|z|<r_{0}\right),
$$

where $r_{0}=r_{0}(p, j, \gamma)$ is given by

$$
r_{0}=r_{0}(p, j, \gamma)=\frac{k-\sqrt{k^{2}-4|2 \gamma-(p-j)|(p-j)}}{2|2 \gamma-(p-j)|},
$$

where $\left(k=2+(p-j)+|2 \gamma-(p-j)|, p \in \mathbb{N}, \gamma \in \mathbb{C}^{*}\right)$.

Remark 2.3. Putting $h(z)=\frac{z^{p}}{1-z}$ in Corollary 2.2, we obtain the result obtained by Altintas and Srivastava [1, Theorem 1].

Putting $h(z)=\frac{z^{p}}{1-z}, A=1, B=-1$ and $j=0$ in Theorem 2.1, we obtain the following result.

Corollary 2.4. Let the function $f \in A(p)$ and suppose that $g \in S_{p}(\gamma)\left(\gamma \in \mathbb{C}^{*}\right)$. If $f(z)$ is majorized by $g(z)$ in $U$, then

$$
\left|f^{\prime}(z)\right| \leq\left|g^{\prime}(z)\right| \quad\left(|z|<r_{0}\right)
$$

where $r_{0}=r_{0}(p ; \gamma)$ is given by

$$
r_{0}=r_{0}(p ; \gamma)=\frac{k-\sqrt{k^{2}-4 p|2 \gamma-p|}}{2|2 \gamma-p|},
$$


where $\left(k=2+p+|2 \gamma-p|, p \in \mathbb{N}, \gamma \in \mathbb{C}^{*}\right)$.

Putting $h(z)=\frac{z^{p}}{1-z}, A=1, B=-1, p=1$ and $j=0$ in Theorem 2.1, we obtain the following result.

Corollary 2.5([2,6]). Let the function $f \in A$ and suppose that $g \in S(\gamma)\left(\gamma \in \mathbb{C}^{*}\right)$. If $f(z)$ is majorized by $g(z)$ in $U$, then

$$
\left|f^{\prime}(z)\right| \leq\left|g^{\prime}(z)\right| \quad\left(|z|<r_{0}\right),
$$

where $r_{0}=r_{0}(\gamma)$ is given by

$$
r_{0}=r_{0}(\gamma)=\frac{k-\sqrt{k^{2}-4|2 \gamma-1|}}{2|2 \gamma-1|},
$$

where $\left(k=3+|2 \gamma-1|, \gamma \in \mathbb{C}^{*}\right)$.

Putting $\gamma=1$ in Corollary 2.5, we obtain the following result.

Corollary 2.6([6,7]) Let the function $f \in A$ and suppose that $g \in S^{*}$. If $f(z)$ is majorized by $g(z)$ in $U$, then

$$
\left|f^{\prime}(z)\right| \leq\left|g^{\prime}(z)\right| \quad\left(|z|<r_{0}\right),
$$

where $r_{0}$ is given by

$$
r_{0}=2-\sqrt{3} .
$$

Using Lemma 2.3 it is easy to prove the following lemma.

Lemma 2.7. Let $\gamma \in \mathbb{C}^{*}$ and $f \in K_{p}^{j}(h ; \gamma)$. Then $f \in S_{p}^{j}\left(h ; \frac{1}{2} \gamma\right)$, that is,

$$
K_{p}^{j}(h ; \gamma) \subset S_{p}^{j}\left(h ; \frac{1}{2} \gamma\right)\left(\gamma \in \mathbb{C}^{*}\right) .
$$

By using Lemma 2.7 and Corollary 2.2, we can prove the following theorem.

Theorem 2.8. Let the function $f \in A(p)$ and suppose that $(g * h) \in K_{p}^{j}(h, \gamma)$. If $(f * h)^{(j)}(z)$ is majorized by $(g * h)^{(j)}(z)$ in $U$, then

$$
\left|(f * h)^{(j+1)}(z)\right| \leq\left|(g * h)^{(j+1)}(z)\right| \quad\left(|z|<r_{0}\right),
$$

where $r_{0}=r_{0}(p, \gamma, j)$ is given by

$$
r_{0}=r_{0}(p, \gamma, j)=\frac{k-\sqrt{k^{2}-4(p-j)|\gamma-(p-j)|}}{2|\gamma-(p-j)|},
$$

where $\left(k=2+(p-j)+|\gamma-(p-j)|, p \in \mathbb{N}, j \in \mathbb{N}_{0}, \gamma \in \mathbb{C}^{*}\right)$. 
Proof. In view of the inclusion property (2.13), Theorem 2.8 can be deduced as a simple consequence of Theorem 2.1 with $\gamma \rightarrow \frac{1}{2} \gamma$.

Remark 2.9. Putting $h(z)=\frac{z^{p}}{1-z}$ in Theorem 2.8, we obtain the result obtained by Altintas [1, Theorem 2].

Putting $h(z)=\frac{z^{p}}{1-z}$ and $j=0$ in Theorem 2.8, we obtain the following result.

Corollary 2.10. Let the function $f \in A(p)$ and suppose that $g \in K_{p}(\gamma)\left(\gamma \in \mathbb{C}^{*}\right)$. If $f(z)$ is majorized by $g(z)$ in $U$, then

$$
\left|f^{\prime}(z)\right| \leq\left|g^{\prime}(z)\right| \quad\left(|z|<r_{0}\right),
$$

where $r_{0}=r_{0}(p ; \gamma)$ is given by

$$
r_{0}=r_{0}(p ; \gamma)=\frac{k-\sqrt{k^{2}-4 p|\gamma-p|}}{2|\gamma-p|},
$$

where $\left(k=2+p+|\gamma-p|, p \in \mathbb{N}, \gamma \in \mathbb{C}^{*}\right)$. result.

Putting $h(z)=\frac{z^{p}}{1-z}, p=1$ and $j=0$ in Theorem 2.8, we obtain the following

Corollary 2.11([1]). Let the function $f \in A$ and suppose that $g \in K(\gamma)$. If $f(z)$ is majorized by $g(z)$ in $U$, then

$$
\left|f^{\prime}(z)\right| \leq\left|g^{\prime}(z)\right| \quad\left(|z|<r_{0}\right),
$$

where $r_{0}=r_{0}(\gamma)$ is given by

$$
r_{0}=r_{0}(\gamma)=\frac{k-\sqrt{k^{2}-4|\gamma-1|}}{2|\gamma-1|}
$$

where $\left(k=3+|\gamma-1|, \gamma \in \mathbb{C}^{*}\right)$.

Letting $\gamma \rightarrow 1$ in Corollary 2.11, we obtain the following result.

Corollary 2.12([7]). Let the function $f \in A$ and suppose that $g \in K$. If $f(z)$ is majorized by $g(z)$ in $U$, then

$$
\left|f^{\prime}(z)\right| \leq\left|g^{\prime}(z)\right| \quad\left(|z| \leq \frac{1}{3}\right) .
$$

\section{Examples}

(i) Putting in (1.6) and (1.7) $h(z)=z^{p}+\sum_{k=p+1}^{\infty} \Gamma_{k-p}\left(\alpha_{1}\right) z^{k}$, (see [4]), where $\Gamma_{k-p}\left(\alpha_{1}\right)=\frac{\left(\alpha_{1}\right)_{k-p} \ldots\left(\alpha_{q}\right)_{k-p}}{\left(\beta_{1}\right)_{k-p} \ldots\left(\beta_{s}\right)_{k-p}(1)_{k-p}}, q \leq s+1, q, s \in \mathbb{N}_{0}, \alpha_{i} \in \mathbb{C}(i=1,2, \ldots, q)$ and 
$\beta_{j} \in \mathbb{C} \backslash \mathbb{Z}_{0}^{-}(j=1,2, \ldots, s)$, then $(f * h)(z)=H_{p, q, s}\left(\alpha_{1}\right) f(z), S_{p}^{j}(h ; \gamma)=S_{p, q, s}^{j}\left(\alpha_{1} ; \gamma\right)$ and $K_{p}^{j}(h ; \gamma)=K_{p, q, s}^{j}\left(\alpha_{1} ; \gamma\right)$. Then we obtain the following results.

Let the function $f \in A(p)$ and $g \in S_{p, q, s}^{j}\left(\alpha_{1} ; \gamma\right)\left(\left|2 \gamma-\alpha_{1}\right|<\left|\alpha_{1}\right|\right)$. If $\left(H_{p, q, s}\left(\alpha_{1}\right) f(z)\right)^{(j)}$ is majorized by $\left(H_{p, q, s}\left(\alpha_{1}\right) g(z)\right)^{(j)}$ in $U$, then

$$
\left|\left(H_{p, q, s}\left(\alpha_{1}+1\right) f(z)\right)^{(j)}\right| \leq\left|\left(H_{p, q, s}\left(\alpha_{1}+1\right) g(z)\right)^{(j)}\right| \quad\left(|z|<r_{1}\right),
$$

where $r_{1}=r_{1}\left(\gamma, \alpha_{1}\right)$ is given by

$$
r_{1}=r_{1}\left(\gamma, \alpha_{1}\right)=\frac{k-\sqrt{k^{2}-4\left|2 \gamma-\alpha_{1}\right|\left|\alpha_{1}\right|}}{2\left|2 \gamma-\alpha_{1}\right|}
$$

where $\left(k=2+\left|\alpha_{1}\right|+\left|2 \gamma-\alpha_{1}\right| ; \gamma, \alpha_{1} \in \mathbb{C}^{*}\right)$.

Also, let the function $f \in A(p)$ and $g \in K_{p, q, s}^{j}\left(\alpha_{1} ; \gamma\right)\left(\left|\gamma-\alpha_{1}\right|<\left|\alpha_{1}\right|\right)$. If $\left(H_{p, q, s}\left(\alpha_{1}\right) f(z)\right)^{(j)}$ is majorized by $\left(H_{p, q, s}\left(\alpha_{1}\right) g(z)\right)^{(j)}$ in $U$, then

$$
\left|\left(H_{p, q, s}\left(\alpha_{1}+1\right) f(z)\right)^{(j)}\right| \leq\left|\left(H_{p, q, s}\left(\alpha_{1}+1\right) g(z)\right)^{(j)}\right|\left(|z|<r_{2}\right),
$$

where $r_{2}=r_{2}\left(\gamma, \alpha_{1}\right)$ is given by

$$
r_{2}=r_{2}\left(\gamma, \alpha_{1}\right)=\frac{k-\sqrt{k^{2}-4\left|\gamma-\alpha_{1}\right|\left|\alpha_{1}\right|}}{2\left|\gamma-\alpha_{1}\right|},
$$

where $\left(k=2+\left|\alpha_{1}\right|+\left|\gamma-\alpha_{1}\right| ; \gamma, \alpha_{1} \in \mathbb{C}^{*}\right)$.

By specializing the parameters $p, q, s, \alpha_{i}(i=1,2, \ldots, q)$ and $\beta_{j}(j=1,2, \ldots, s)$, we obtain various results for different operators.

(ii) Putting in (1.6) and (1.7) $h(z)=z^{p}+\sum_{k=p+1}^{\infty}\left[\frac{p+\ell+\lambda(k-p)}{p+\ell}\right]^{m} z^{k}$, (see, [3]), where $\ell \geq 0, \lambda>0, m \in \mathbb{N}_{0}$, then $(f * h)(z)=I_{p}^{m}(\lambda, \ell) f(z), S_{p}^{j}(h ; \gamma)=S_{p}^{m, j}(\lambda, \ell ; \gamma)$ and $K_{p}^{j}(h ; \gamma)=K_{p}^{m, j}(\lambda, \ell ; \gamma)$. Also, we obtain the following results.

Let the function $f \in A(p)$ and $g \in S_{p}^{m, j}(\lambda, \ell ; \gamma)\left(\left|2 \gamma-\left(\frac{p+\ell}{\lambda}\right)\right|<\left(\frac{p+\ell}{\lambda}\right)\right)$. If $\left(I_{p}^{m}(\lambda, \ell) f(z)\right)^{(j)}$ is majorized by $\left(I_{p}^{m}(\lambda, \ell) g(z)\right)^{(j)}$ in $U$, then

$$
\left|\left(I_{p}^{m+1}(\lambda, \ell) f(z)\right)^{(j)}\right| \leq\left|\left(I_{p}^{m+1}(\lambda, \ell) g(z)\right)^{(j)}\right|\left(|z|<r_{3}\right),
$$

where $r_{3}=r_{3}(p, \gamma, \lambda, \ell)$ is given by

$$
r_{3}=r_{3}(p, \gamma, \lambda, \ell)=\frac{k-\sqrt{k^{2}-4\left|2 \gamma-\left(\frac{p+\ell}{\lambda}\right)\right|\left(\frac{p+\ell}{\lambda}\right)}}{2\left|2 \gamma-\left(\frac{p+\ell}{\lambda}\right)\right|},
$$

where $\left(k=2+\left(\frac{p+\ell}{\lambda}\right)+\left|2 \gamma-\left(\frac{p+\ell}{\lambda}\right)\right|, p \in \mathbb{N}, \gamma \in \mathbb{C}^{*}, \ell \geq 0, \lambda>0, m \in \mathbb{N}_{0}\right)$. 
Also, let the function $f \in A(p)$ and $g \in K_{p}^{m, j}(\lambda, \ell ; \gamma)\left(\left|\gamma-\left(\frac{p+\ell}{\lambda}\right)\right|<\left(\frac{p+\ell}{\lambda}\right)\right)$. If $\left(I_{p}^{m}(\lambda, \ell) f(z)\right)^{(j)}$ is majorized by $\left(I_{p}^{m}(\lambda, \ell) g(z)\right)^{(j)}$ in $U$, then

$$
\left|\left(I_{p}^{m+1}(\lambda, \ell) f(z)\right)^{(j)}\right| \leq\left|\left(I_{p}^{m+1}(\lambda, \ell) g(z)\right)^{(j)}\right| \quad\left(|z|<r_{4}\right),
$$

where $r_{4}=r_{4}(p, \gamma, \lambda, \ell)$, is given by

$$
r_{4}=r_{4}(p, \gamma, \lambda, \ell)=\frac{k-\sqrt{k^{2}-4\left|\gamma-\left(\frac{p+\ell}{\lambda}\right)\right|\left(\frac{p+\ell}{\lambda}\right)}}{2\left|\gamma-\left(\frac{p+\ell}{\lambda}\right)\right|},
$$

where $\left(k=2+\left(\frac{p+\ell}{\lambda}\right)+\left|\gamma-\left(\frac{p+\ell}{\lambda}\right)\right|, p \in \mathbb{N}, \gamma \in \mathbb{C}^{*}, \ell \geq 0, \lambda>0\right)$.

By specializing the parameters $p, \lambda, \ell$ and $m$ we obtain various results for different operators.

Acknowledgements The authors thank the referees for their valuable suggestions which led to improvement of this paper.

\section{References}

[1] O. Altintas and H. M. Srivastava, Some majorization properties associated with p-valent staslike and convex functions of complex order, East Asian Math. J., $\mathbf{1 7 ( 2 ) ( 2 0 0 1 ) , ~ 1 7 5 - 1 8 3 . ~}$

[2] O. Altintas, O. Ozkan and H. M. Srivastava, Majorization by starlike functions of complex order, Complex Var., 46(2001), 207-218.

[3] A. Catas, On certain classes of p-valent functions defined by multiplier transformations, in Proceedings of the International Symposium on Geometric Function Theory and Applications: GFTA 2007 Proceedings (İstanbul, Turkey; 20-24 August 2007) (S. Owa and Y. Polatoglu, Editors), pp. 241-250, TC .Istanbul Kültűr University Publications, Vol. 91, TC .Istanbul Kültür University, İstanbul, Turkey, 2008.

[4] J. Dziok and H. M. Srivastava, Classes of analytic functions associated with the generalized hypergeometric function, Appl. Math. Comput., 103(1999), 1-13.

[5] A. W. Goodman, On the Schwarz-Christoffel transformation and p-valent functions, Trans. Math. Soc., 68(1950), 204-223.

[6] S. P. Goyal and P. Goswami, Majorization for certain classes of analytic functions defined by fractional derivatives, Appl. Math. Letters, 22(2009), 1855-1858.

[7] T. H. MacGregor, Majorization by univalent functions, Duke Math. J., 34(1967), 95-102.

[8] S. S. Miller and P. T. Mocanu, Differential Subordinations: Theory and Applications, Series on Monographs and Textbooks in Pure and Applied Mathematics, Vol. 225, Marcel Dekker Inc., New York. and Basel, 2000. 
[9] M. A. Nasr and M. K. Aouf, Starlike function of complex order, J. Nature. Sci. Math., 25(1985), 1-12.

[10] Z. Nehari, Conformal Mapping, MacGraw-Hill Book Company, New York, Toronto and London 1952.

[11] S. Owa, On certain classes of p-valent functions with negative coefficients, Simon Stevin, 59(1985), 385-402.

[12] D. A. Patil and N. K. Thakare, On convex hulls and extreme points of p-valent starlike and convex classes with applications, Bull. Math. Soc. Math. R. S. Roumaine (N. S.), 27(1983), no.75, 145-160.

[13] P. Wiatrowski, On the coefficients of some of some family of holomorphic functions, Zeszyry Nauk. Univ. Lddz. Nauk. Mat.-Przyrod., 30(1970), 75-85. 